\title{
Computational Study on the Function of Palmitoylation on the Envelope Protein in SARS-CoV-2
}

\author{
Shengjie Sun, Chitra Karki, Javier Aguilera, Alan E. Lopez Hernandez, Jianjun Sun, and Lin Li*
}

Cite This: https://doi.org/10.1021/acs.jctc.1c00359

Read Online

\section{ACCESS \\ 山ll Metrics \& More \\ Article Recommendations \\ Supporting Information}

ABSTRACT: SARS-CoV-2 that caused COVID-19 has spread since the end of 2019. Its major effects resulted in over four million deaths around the whole world by August 2021. Therefore, understanding virulence mechanisms is important to prevent future outbreaks and for COVID-19 drug development. The envelope (E) protein is an important structural protein, affecting virus assembly and budding. The E protein pentamer is a viroporin, serving as an ion transferring channel in cells. In this work, we applied molecular dynamic simulations and topological and electrostatic analyses to study the effects of palmitoylation on the $\mathrm{E}$ protein pentamer. The results indicate that the cation transferring direction is more from the lumen to the cytosol. The structure of the palmitoylated $\mathrm{E}$ protein pentamer is more stable while the loss of palmitoylation caused the pore radius

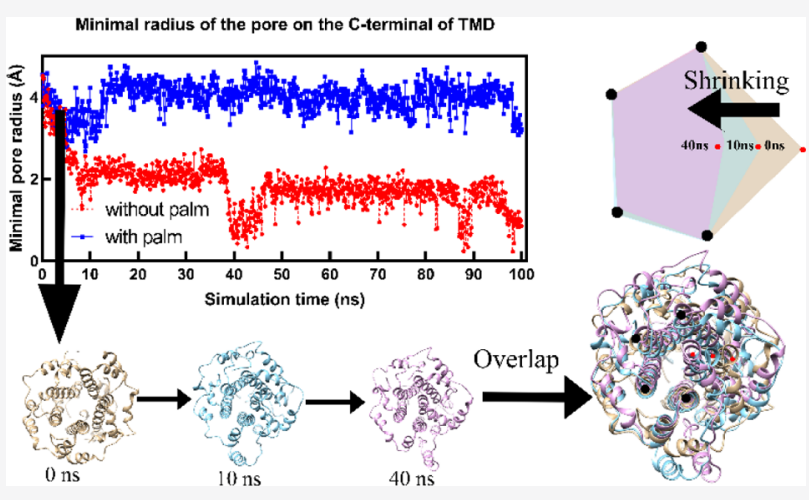
to reduce and even collapse. The electrostatic forces on the two sides of the palmitoylated $\mathrm{E}$ protein pentamer are more beneficial to attract cations in the lumen and to release cations into the cytosol. The results indicate the importance of palmitoylation, which can help the drug design for the treatment of COVID-19.

\section{INTRODUCTION}

Coronaviruses (CoVs) are notorious as the pathogens of numerous diseases in a wide range of vertebrates, including human beings. In 2002, the SARS-CoV-related severe acute respiratory syndrome (SARS) took 4 months to overwhelm 29 countries and killed 774 reported patients. It almost paralyzed the Asian economy. ${ }^{1}$ After 10 years, the outbreak of a new coronavirus, named the Middle East respiratory syndrome coronavirus (MERS-CoV), spread in 27 countries, causing a $35 \%$ death rate. ${ }^{1 \mathrm{~b}, 2}$ In December 2019, a novel coronavirus named severe acute respiratory syndrome coronavirus 2 (SARS-CoV-2) was discovered and now has already spread across the whole world. The disease caused by it is known as coronavirus disease 2019 (COVID-19). ${ }^{3}$ So far, the disease has infected 106 million people and caused over 4 million deaths in the whole world. ${ }^{4}$ In addition to severe pneumonia, the SARSCoV-2 can also cause multi-organ damage, including cardiovascular disease, ${ }^{5}$ reproductive risk, ${ }^{6}$ mental illness, and smell disfunction. ${ }^{8}$ Thus, it is urgent to understand the virulence mechanisms to prevent future outbreaks and to develop the remedies for COVID-19.

The SARS-CoV-2 is a positive-RNA strand virus that belongs to the family Coronaviridae. ${ }^{3 a, 9}$ The genome of coronavirus encodes four major structural proteins, which are the spike $(\mathrm{S})$ protein, nucleocapsid $(\mathrm{N})$ protein, membrane (M) protein, and envelope (E) protein. ${ }^{10}$ The $S$ protein mainly regulates the binding with the ACE2 (angiotensin-converting enzyme) receptor of cells. ${ }^{11}$ The $\mathrm{N}$ and the $\mathrm{M}$ protein serve as
RNA genome-binding protein ${ }^{12}$ and the most abundant structural protein of the viral envelope, respectively. ${ }^{13}$ The E protein is the smallest among four major structural proteins. Other than the $\mathrm{S}, \mathrm{N}$, and $\mathrm{M}$ proteins, the $\mathrm{E}$ protein is abundantly expressed in the infected cells but only a small percentage is assembled into the viral envelope. ${ }^{14}$ The majority of the protein is located on the ER (endoplasmic reticulum), Golgi complex, and ERGIC (ER-Golgi intermediate compartment). ${ }^{15}$ The E protein is related to intracellular trafficking and participates in the viral assembly and budding. ${ }^{14,16}$ The recombined $\mathrm{CoVs}$ which lack $\mathrm{E}$ protein express crippled viral maturation and incompetent progeny. ${ }^{17}$ The study about the $\mathrm{E}$ protein is important to understand virulence mechanisms.

The SARS-CoV E protein contains a short hydrophilic terminal, a large hydrophobic transmembrane domain (TMD), and a long hydrophilic carboxyl end (Figure S2). ${ }^{18}$ Synthetic peptides of the TMD of E protein can form dimers, trimers, and pentamers, ${ }^{19}$ among which the pentametric structure is widely accepted and studied as a viroporin. ${ }^{13 a, 14,15}$ Similarly, the TMD of SARS-CoV-2 E protein was also found to form a

Received: April 11, 2021 
five-helix buddle surrounding a narrow pore. ${ }^{19}$ The narrow pore is suggested as a viroporin to regulate multiple stages of viral life circles. Viroporins can transport different ions but usually prefer cations $\left(\mathrm{H}^{+}, \mathrm{K}^{+}, \mathrm{Na}^{+}\right.$, and $\left.\mathrm{Ca}^{2+}\right) .{ }^{20}$ As reported, the cell infected with coronavirus exhibited a remarkable increase in $\mathrm{pH}$ in the Golgi complex. The increased $\mathrm{pH}$ protects spike protein and promotes the release of the virus from the cell. ${ }^{16}$ This finding supports that the $\mathrm{E}$ protein pentamer of coronavirus could be a $\mathrm{H}^{+}$channel, transferring $\mathrm{H}^{+}$from lumen to cytosol. The higher $\mathrm{pH}$ in the lumen may help in release of the virus. To modulate protein functions, post-translational modifications (PTMs) are necessary. The PTMs of the E protein of coronavirus include glycosylation and palmitoylation. ${ }^{13 a, 21} \mathrm{~N}$-glycosylation, as reported, appears in a minor form of SARS-CoV E protein, which has both Cand N-terminals exposed on the luminal side. ${ }^{22}$ Nevertheless, the $\mathrm{E}$ protein with both the $\mathrm{C}$ - and $\mathrm{N}$-terminal on the cytoplasmic side is not modified by glycosylation. By contrast, all three cysteine residues (C40, C43, and C44) in the SARS$\mathrm{CoV} \mathrm{E}$ protein are modified by palmitoylation. ${ }^{23}$ The mutants of these cysteine residues in $\mathrm{E}$ protein of mouse hepatitis virus A59 (MHV-A59) weaken the ability to form virus-like particles. $^{24}$ Additionally, MHV was prone to degradation when carrying triple mutants. ${ }^{24 a}$ Additionally, the palmitoylated protein with higher hydrophobicity benefits membrane anchoring and association. ${ }^{25}$ The studies on human coronavirus also proved the poor localization of nonpalmitoylated E protein in membranes, while the palmitoylated E proteins are not affected. ${ }^{24 a}$ These findings proved the importance of palmitoylation on the $\mathrm{E}$ protein.

To better understand how palmitoylation affects the function of coronavirus E protein, the SARS-CoV-2 E protein pentameric structure was used as the reference (E protein without palmitoylation). The palmitoylation was performed on the cysteine residues 40,43 , and 44 to each monomer as the palmitoylated $\mathrm{E}$ protein pentamer (E protein with palmitoylation). Afterward, molecular dynamics (MD) simulations were run to observe the effects of palmitoylation. Besides, the topological and electrostatic studies were applied to analyze the function of palmitoylation. In the end, we simulated several layers of $\mathrm{H}^{+}$ions on two sides to detect the differences in the force on cations. These studies were summarized in the Conclusions section to discuss the effects of palmitoylation on the function of passing ions on the $\mathrm{E}$ protein pentamer.

\section{METHOD}

2.1. Modeling and Simulation. 2.1.1. Modeling. The sequence of E protein monomer of SARS-CoV-2 from 2019 was used as the query sequence (RefSeq: YP_009724392.1) (Figure S1). The three-dimensional pentameric structure of the E protein of the SARS-CoV-2 structure was based on the pentameric structure of the SARS-CoV E protein of the PDB $5 \times 29^{26}$ and was achieved using the Robetta server. ${ }^{27}$ Figure S1 shows the sequence and structural alignment of SARS-CoV-2 and SARS-CoV. The sequence similarity is $96 \%$. The modeled pentameric structure was used as the initial configuration ( $\mathrm{E}$ protein without palmitoylation) for the MD simulation. The palmitoylation was performed on the cysteine residues 40,43 , and 44 of each monomer (Figure 1) to yield the E protein pentamer with palmitoylation. The palmitate groups were added using CHARMM-GUI ${ }^{28}$ by CHARMM36 force field. ${ }^{29}$

2.1.2. Simulation. The CHARMM-GUI ${ }^{28}$ webserver was used to create the simulating system (Figure 1). The two

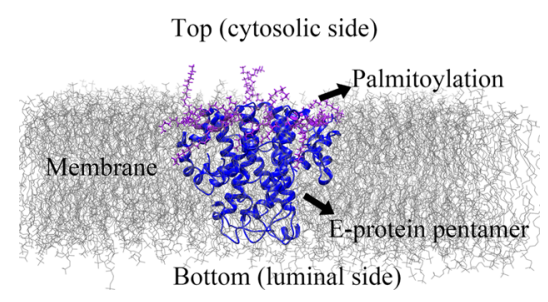

Figure 1. Diagram of the E protein pentamer in the membrane. The $\mathrm{C}$-terminal of the $\mathrm{E}$ protein pentamer is on the top, cytosolic side, and the $\mathrm{N}$-terminal of the $\mathrm{E}$ protein pentamer is on the bottom, luminal side. The membrane lipid is represented in gray lines. The $\mathrm{E}$ proteins are represented in the carton (blue). The palmitate groups (palmitoylation) are colored purple.

structures were then embedded in the membrane dioleoyl phosphatidylcholine (DOPC) around the center along the $Z$ axis. The DOPC is used widely in experiments close to the membrane where E protein is found. ${ }^{30}$ The membrane is a square with a side length of $150 \AA$ along the $x$-and $y$-axis. The system was solvated with water of type TIP $3 \mathrm{P}^{31}$ of thickness $15 \AA$ on either side of the membrane. $\mathrm{NaCl}$ was used to ionize the system with a concentration of $150 \mathrm{mM}$. Parameterization of the atoms in the system was attained with the CHARMM36 force field. ${ }^{29}$ Periodic boundary condition was applied to the simulating box, and particle mesh Ewald ${ }^{32}$ was used for the long-range electrostatic interactions. The final system was then subjected to the MD simulation with NAMD 2.12. ${ }^{33}$ The whole simulation includes two steps. The first is equilibration, and the second is production run. NPT was used for the equilibration. The temperature was set to $300 \mathrm{~K}$ and the pressure was set to $1 \mathrm{~atm}$ using a Langevin thermostat with a damping coefficient of $1 /$ ps and a Nosé-Hoover Langevin piston barostat with a decay period of $25 \mathrm{fs}$, respectively. The temperature was reassigned every 500 steps. During the equilibration, a constraint was applied to the entire $\mathrm{E}$ protein pentamer with palmitoylation and the headgroup of the DOPC lipid. In the production run, NPT ensemble was continued for $100 \mathrm{~ns}$. The constraints on E proteins and headgroups of the DOPC membrane were released during the production run. For nonbonded interactions (electrostatic and van der Waal), the cutoff was set to $12 \AA$. The switching distance was set to 10 A. In the production run, the NPT ensemble was continued for100 ns. The constraints on E proteins and headgroups of the DOPC membrane were released during the production run. $\mathrm{E}$ protein pentamers with and without palmitoylation simulations were repeated two more times. The analyses were based on the production runs.

2.2. Topological Study. As shown in Figures S1 and S2, the TMD of the E protein pentamer is marked from $17 \mathrm{~V}$ to 37L ( 21 residues) in five monomers for RMSF (root-meansquare fluctuations) analysis based on the stable state (60-100 $\mathrm{ns})$. The diagram (Figure 1) shows that the cytosolic side (Cterminal) is on the top while the luminal side ( $\mathrm{N}$-terminal) is on the bottom. ${ }^{13 a}$

2.2.1. RMSF and Root-Mean-Square Deviation. The RMSF of the $\alpha$-carbons of the residues (21 residues) on the TMD is achieved based on the last $40 \mathrm{~ns}$ simulations by visual MDs (VMDs) ${ }^{34}$ (eq 1$) .^{35}$

$$
\mathrm{RMSF}_{\mathrm{i}}=\left[\frac{1}{T} \sum_{t_{\mathrm{j}}=1}^{T}\left|r_{\mathrm{i}}\left(t_{\mathrm{j}}\right)-r_{\mathrm{i}}^{\mathrm{ref}}\right|\right]^{1 / 2}
$$


where $\mathrm{i}$ represents the residue ID, $T$ represents the total simulation time (here is the number of frames), $r_{\mathrm{i}}\left(t_{\mathrm{j}}\right)$ represents the residue $\mathrm{i}$ in the time of $t_{\mathrm{j}}$ position, and $r_{\mathrm{i}}^{\text {ref }}$ is the reference position of the residue $i$, calculated from the time-average position.

The RMSD (root-mean-square deviation) is to measure the average distance between two protein structures, calculated using eq $2^{34,35}$

$$
\operatorname{RMSD}(t)=\left[\frac{1}{W N} \sum_{\mathrm{i}=1}^{N} w_{\mathrm{i}}\left|r_{\mathrm{i}}(t)-r_{\mathrm{i}}^{\mathrm{ref}}\right|^{2}\right]^{1 / 2}
$$

where $W=\Sigma w_{\mathrm{i}}$ is the weighting factor, $N$ is the total number of atoms, $r_{\mathrm{i}}(t)$ is the position of atom $\mathrm{i}$ at time $t$ after least square fitting the structure to the reference structure, and $r_{i}^{\text {ref }}$ is the reference position of residue $i$ defined by the reference structure (here we used the initial structure as the reference).

2.2.2. General Comparison of the Projections of the $E$ Protein Pentamer's Top and Bottom Views. The E protein pentamers with and without palmitoylation were compared to show the conformational differences in both top and bottom views. The structures were from the last frame (at $100 \mathrm{ns)} \mathrm{of}$ the simulation.

2.2.3. Minimal Pore Radius and Pore Volume Testing. The pore radius is tested using HOLE $2^{36}$ for the $\mathrm{E}$ protein pentamer with and without palmitoylation in 60-100 ns simulations. The minimal pore radius is the minimum radius of the ion channel formed by the E protein pentamer TMD. To avoid the spherical probe ball running in the wrong direction, the moving direction is restrained to follow the $Z$-axis. ${ }^{36}$ The pore radius was tested three times for a single frame, and the maximum value was taken as the minimal pore radius. It is because of the existence of a gradient when HOLE increases the probe size. To better understand the effect of palmitoylation on channel size, the TMD was split into three parts, including the C-terminal part (residue 30-39), middle part (residue 20-30), and N-terminal part (residue 11-20), to test the minimal radius separately. The volume is calculated by integration through the pore.

2.2.4. Electrostatic Potential Study. The structures of the palmitoylated and non-palmitoylated E protein pentamer were from the last frame (at $100 \mathrm{~ns}$ ) of the simulations. The electrostatic potential calculations of the $\mathrm{E}$ protein pentamer were carried out using Delphi. ${ }^{37}$ The charge and the radius of atoms were calculated using the force field CHARMM36 and assigned using PDB2PQR. ${ }^{38}$ The dielectric constants were set as 2 for proteins and membranes, ${ }^{39}$ while 80 for water. The salt concentration was set as $150 \mathrm{mM}$, and the probe radius was set as $1.4 \AA$. The protein filling percentage was set as $70 \%$, and the resolution was set as 1.5 grids/Å. The electrostatic potentials on the surfaces were visualized using Chimera ${ }^{40}$ for both cytosolic (top view) and luminal sides (bottom view). The color range was set from $-1.0 \mathrm{kT} / \mathrm{e}$ (red) to $1.0 \mathrm{kT} / \mathrm{e}$ (blue). The potential surfaces were visualized using Chimera, ${ }^{40}$ and the electric field lines were visualized using $\mathrm{VMD}^{34}$ to demonstrate the interactions.

2.2.5. Principal Component Analysis. The mass centers of the TMD from 60-100 ns (1000 frames) were selected for principal component analysis (PCA). The $X, Y$, and $Z$ of the mass center were treated as variables to perform PCA. The analyses were applied separately for the non-palmitoylated $\mathrm{E}$ protein and palmitoylated E protein.
2.2.6. Electrostatic Force Study. To quantitatively demonstrate the electrostatic force on the cations along with the distance with the membrane, $\mathrm{H}^{+}$ions were selected. On both cytosolic and luminal sides, ten spherical layers of $\mathrm{H}^{+}$ions were simulated (Figure S3). The nearest layer is $1 \AA$ away from the membrane while the farthest is $10 \AA$. For each layer, there are $288 \mathrm{H}^{+}$ions; the center is on the $Z$-axis, which passed the mass center of the TMD. In each layer, the radiuses of $\mathrm{H}^{+}$circles are from 3 to $10 \AA$. In a certain circle, for every $10^{\circ}$, a hydrogen ion is placed. The electrostatic force on $\mathrm{H}^{+}$was split along the $Z$-axis ( $Z$ component) to demonstrate the component force of the direction. The $Z$ component force direction was set from the bottom (luminal side) to the top (cytosolic side). The electrostatic force was calculated using DelphiForce, ${ }^{41}$ and the parameters were the same as that of potential surface calculations.

\section{RESULT AND DISCUSSION}

3.1. Topological Study. From the RMSD study of the TMD (Figure S4), both systems have achieved stability after $10 \mathrm{~ns}$ simulation. From the RMSD of the repeated simulations, both the E protein pentamer with and without palmitoylation also reached stability after $40 \mathrm{~ns}$. The repeated simulations also show that the E protein pentamer reached stability after $40 \mathrm{~ns}$. The structures in Figure 2 are the $\mathrm{E}$ protein pentamers

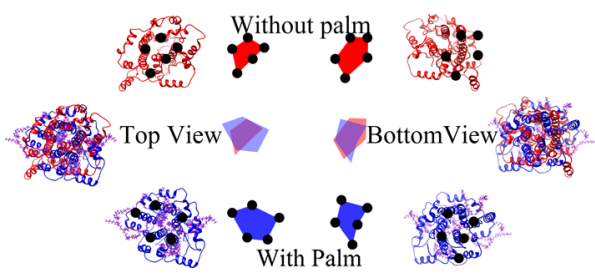

Figure 2. General comparison of the $\mathrm{E}$ protein pentamer in the top and bottom view. The non-palmitoylated $\mathrm{E}$ protein pentamer is red colored while the palmitoylated $\mathrm{E}$ protein pentamer is colored blue. The palmitate groups are colored purple.

extracted from the last frame (at $100 \mathrm{~ns}$ ) of the MD simulations of both systems. The structures were aligned using Chimera, ${ }^{40}$ and the two sides of the ion channel were compared from the top and bottom views. From the general comparison, the palmitoylated $\mathrm{E}$ protein pentamer stabilizes the pentametric structure in the larger pore size on the cytosolic sides while the loss of palmitoylation reduces the pore radius on the cytosolic side and reshapes the structure into an irregular conformation. By contrast, there is no significant difference in the bottom view. In Figure S6, we also compared the structures in the two repeated simulations in the top view (structures are from the frame at $100 \mathrm{~ns}$ ). The comparison shows the same result. To further investigate the effects of palmitoylation on the whole ion channel during simulations, the HOLE2 ${ }^{36}$ program was applied to test the minimal pore radius of the C-terminal part, middle apart, and the N-terminal part of the ion channel.

As shown in Figure $3 \mathrm{~A}$, in the equilibrium state $(60-100$ $\mathrm{ns}$ ), the C-terminal part of the palmitoylated $\mathrm{E}$ protein pentamer exhibits a minimal pore radius of $3.96 \AA$ (standard deviation: $0.31 \AA$ ). By contrast, the C-terminal part of the nonpalmitoylated $\mathrm{E}$ protein pentamer only has a $1.56 \AA$ minimal pore radius (standard deviation: $0.35 \AA$ ). The loss of palmitoylation directly reduces the pore radius. Without 
A Minimal radius of the pore on the C-terminal of TMD
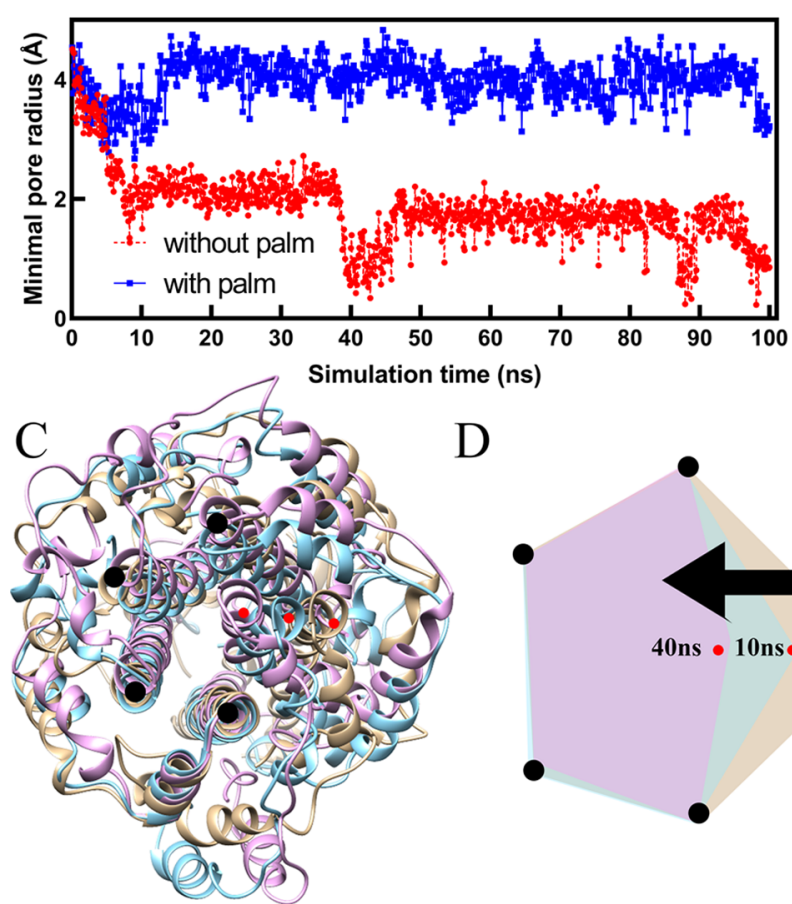

$\mathrm{B}$

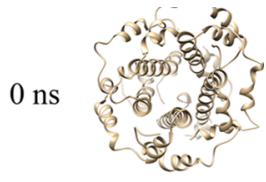

$10 \mathrm{~ns}$

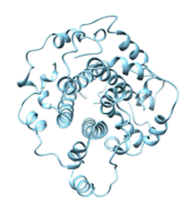

$\mathrm{D}$
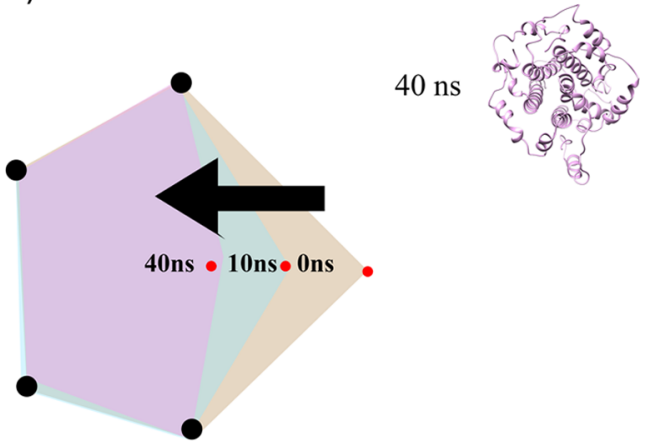

Figure 3. Minimal pore radius of the $\mathrm{E}$ protein pentamer on the $\mathrm{C}$-terminal during the simulation (A) and the structure comparison of the $\mathrm{E}$ protein pentamer without palmitoylation at 0,10 , and $40 \mathrm{~ns}(\mathrm{~B}-\mathrm{D})$.

A

The number of salt bridges of $E$ protein during 50ns simulation
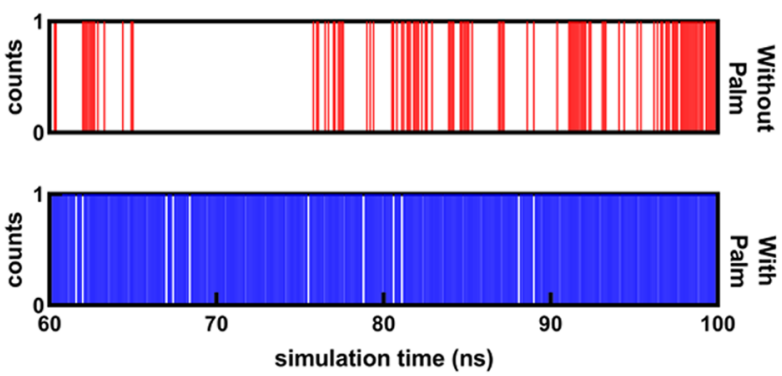

ARG61 Chain E

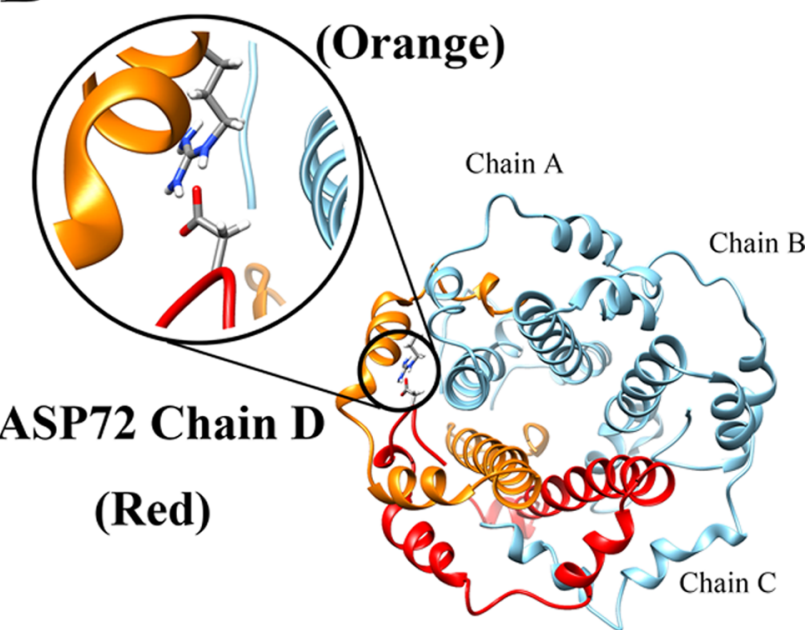

Figure 4. Number of salt bridges in the E-protein pentamer in adjacent monomers with and without palmitoylation during 60-100 ns simulations (A) and the sole salt bridge (ARG61-ASP72) in both the E protein pentamer with and without palmitoylation (B).

palmitoylation, the collapse of the ion channel happened twice at 10 and $40 \mathrm{~ns}$, respectively. The structures (Figure 3B) were extracted from the simulation at 0,10 , and $40 \mathrm{~ns}$ for structural comparison (Figure 3C). The diagram (Figure 3D) clearly shows one of the helix monomers' movements (red dots) during the simulation, causing the pore radius to shrink. The volume calculated from the C-terminal part of the TMD also shows that the $\mathrm{E}$ protein pentamer with palmitoylation possesses a higher capacity for ion transfer while the loss of palmitoylation makes the capacity of the $\mathrm{E}$ protein pentamer lower (Figure S8). Besides, palmitoylation at the C-terminal also affects the middle part of the TMD (Figure S8). The minimal pore radius in the middle part of the TMD of the palmitoylated $\mathrm{E}$ protein pentamer is larger than that without palmitoylation. Although in the N-terminal part (Figure S9), palmitoylation does not affect. This finding is consistent with the general comparison, which shows a higher area in the top view for the palmitoylated $\mathrm{E}$ protein pentamer but no significant difference in the bottom view.

To investigate the reason for the collapses, the salt bridges and RMSF were calculated for the $\mathrm{E}$ protein pentamers. Interestingly, the salt bridge in both the $\mathrm{E}$ protein pentamer with and without palmitoylation is the same one (ARG61 chain D-ASP72 chain E); the occupancy of this in the E 
protein pentamer with palmitoylation is close to 1 while that in E protein without palmitoylation is around 0.4 (Figure 4). The distance of ARG61(chain D)-ASP72(chain E) is over $3 \AA$, revealing that the salt bridge belongs to the weak electrostatic binding. Due to a salt bridge threshold of $4 \AA$, the residue pairs of ARG61-ASP72 in other adjacent chains are not displayed. We further extend the threshold to calculate the distance of ARG61(chain A)-ASP72(chain B) (Figure S12). The result shows that ARG61(chain A)-ASP72(chain B) in the palmitoylated $\mathrm{E}$ protein pentamer possess a lower distance of $6 \AA$ while that in the non-palmitoylated E protein is over $10 \AA$. It means that the structure of the $E$ protein pentamer without palmitoylation is unstable compared with that of the E protein pentamer with palmitoylation. The RMSF study also shows that the $\alpha$-carbons in the TMD residues of the nonpalmitoylated $\mathrm{E}$ protein pentameric structure are higher than those in the palmitoylated E protein pentamer (Figure S11). Both results reveal the instability of the non-palmitoylated $\mathrm{E}$ protein pentamer. The loss of palmitoylation caused an unstable pentametric structure, leading to the narrow pore radius and collapses of the ion channel. In previous studies, the palmitoylation of the E protein pentamer has also been shown with high importance in murine coronavirus assembling. ${ }^{24 \mathrm{~b}}$ The related palmitoylated residues have also been studied in the previous studies. ${ }^{24 a}$ However, mechanism studies were not applied in them. Unlike previous studies, this work focused on the mechanism of how palmitoylation affects the $\mathrm{E}$ protein pentamer in computational studies. The function of palmitoylation in stabilizing proteins has also been found in rous sarcoma virus (RSV) transmembrane glycoprotein, ${ }^{42}$ CCR5 receptor, ${ }^{43}$ and TEAD proteins. $^{44}$ The mechanism found in this work may be also related to the other palmitoylated transmembrane proteins.

3.2. Electrostatic Study. As shown in Figure 5, the top side (cytosolic side) of the electrostatic surface of the E protein

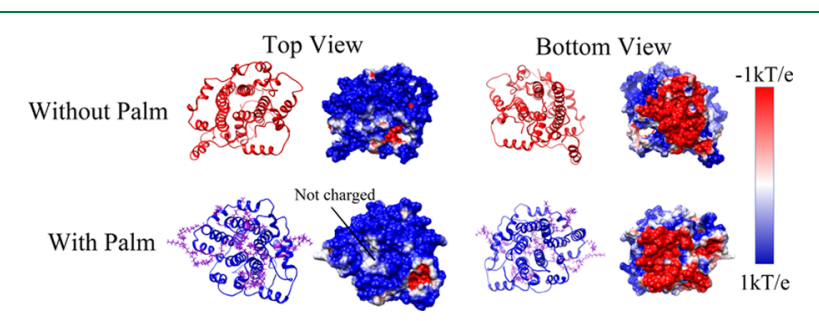

Figure 5. Electrostatic surface of the E protein pentamer with and without palmitoylation in the top and bottom view. The color range was set from $-1.0 \mathrm{kT} / \mathrm{e}$ (red) to $1.0 \mathrm{kT} / \mathrm{e}$ (blue).

pentamer is positively charged while the bottom side (luminal side) of that is negatively charged. This configuration provides that the electrostatic forces acting on cations on two sides are from lumen to cytosol, which is suitable for attracting cation on the luminal side and releasing cation on the cytosolic side. Combined with cation preference ${ }^{20 \mathrm{e}}$ for viroporin, if the cation is $\mathrm{H}^{+}$ions, the release of $\mathrm{H}^{+}$from the lumen to the cytoplasm by the $\mathrm{E}$ protein pentamer would cause higher $\mathrm{pH}$ in the lumen. The result is consistent with the previous finding that the loss of the $\mathrm{E}$ protein pentamer causes a high $\mathrm{pH}$ in the Golgi complex. ${ }^{16}$ From the top view, the center of the $\mathrm{E}$ protein is positively charged (Figure 5). The palmitoylation on the cytosolic side increases the positively charged area. The enlarged area strengthens the repulsive force on the cation on the top side. By contrast, even the luminal side (Figure 5 bottom view) of the E protein pentamer is not palmitoylated; the surface of this does not show significant differences. The electrostatic surface and electric field lines of the membrane with the E protein pentamer are visualized in Figure 6. From

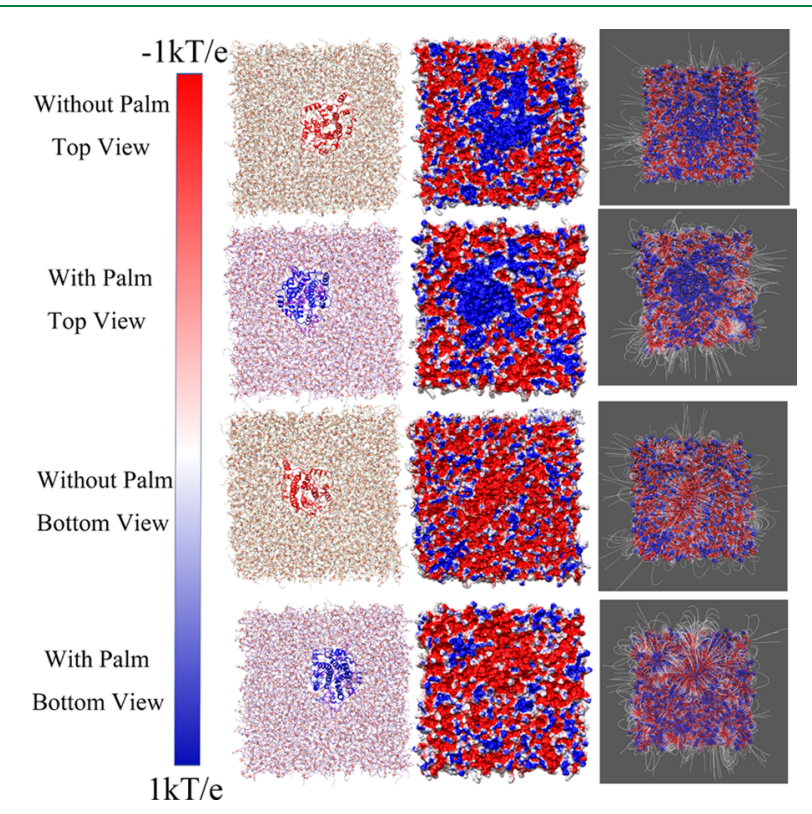

Figure 6. Electrostatic surfaces and electric field lines of the membrane/E protein with and without palmitoylation. The membrane structure (with the $\mathrm{E}$ protein pentamer) is represented in the first column; the electrostatic surfaces are represented in the second column; and the field lines are presented in the third column. The color range was set from $-1.0 \mathrm{kT} / \mathrm{e}$ (red) to $1.0 \mathrm{kT} / \mathrm{e}$ (blue).

the top view, the positively charged area is extended by palmitoylation. It is consistent with the results of the $\mathrm{E}$ protein pentamer in Figure 5. For the bottom view of the membranes, the $\mathrm{E}$ protein pentamers with and without palmitoylation show no significant differences in the electrostatic potential at the surfaces. However, the electric field lines in the $\mathrm{E}$ protein pentamer with palmitoylation show intensive interactions with the surrounding membrane. Compared with findings in Figure 6 , the palmitoylation on the top can enhance the interactions on the bottom.

3.3. Principal Component Analysis. In Figure 6 (first column), the clear movement of the palmitoylated $\mathrm{E}$ protein pentamer is observed. So, the PCA was applied on both the palmitoylated $\mathrm{E}$ protein and non-palmitoylated $\mathrm{E}$ protein pentamer separately. The results (Figure S13) show that in four stages (stage 1: $60-70 \mathrm{~ns}$, stage 2 : $70-80 \mathrm{~ns}$, stage 3: $80-$ $90 \mathrm{~ns}$, and stage 4: 90-100 ns), the center of the nonpalmitoylated $\mathrm{E}$ protein pentamer moved based on one center, while the palmitoylated $\mathrm{E}$ protein pentamer shows a clear motion trajectory in four stages. This finding infers that the palmitoylated $\mathrm{E}$ protein pentamer possesses higher motility in the membrane.

3.4. Electrostatic Force on $\mathbf{H}^{+}$. To better analyze the electrostatic force on the cations when the $\mathrm{E}$ protein pentamer is approached, several layers of $\mathrm{H}^{+}$ions (along the $Z$-axis) were simulated to be placed on both sides of the membrane (Figure S3). The farthest layer of $\mathrm{H}^{+}$was $10 \AA$ away from the membrane. The electrostatic forces were split into the $X, Y$, and $Z$ components. The $Z$ component is the main force to attract or repel ions. On the cytosolic side (Figure 7A), the $Z$ 


\section{A The Z-component of the electrostatic foce on the simulated $\mathrm{H}^{+}$on the $\mathrm{C}$-terminal}

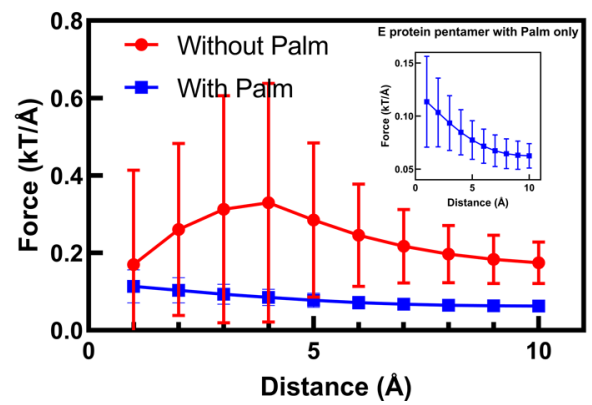

$\mathrm{B}$ The Z-component of the electrostatic foce on the simulated $\mathrm{H}^{+}$on the $\mathrm{N}$-terminal

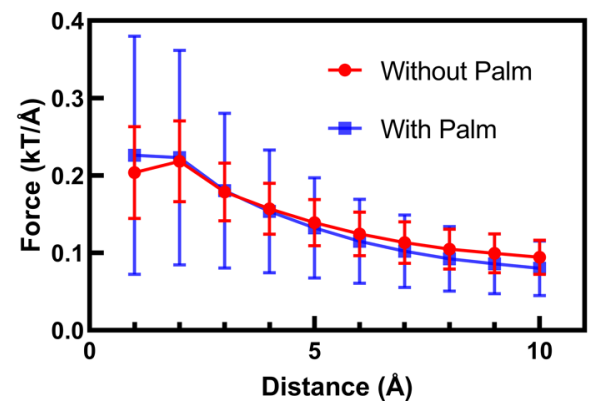

Figure 7. $\mathrm{Z}$ component of the electrostatic force on $\mathrm{H}^{+}$on both C-terminal (A) and $\mathrm{N}$-terminal (B) sides of the E-protein pentamer. The distance is the distance with the membrane. The error bars represent the standard deviation.

component of the electrostatic force shows a constant decrease on the palmitoylated $\mathrm{E}$ protein pentamer when the $\mathrm{H}^{+}$leaving the membrane and the direction is from lumen to cytoplasm. By contrast, the loss of the palmitoylation resulted in nonconstant $Z$ components when $\mathrm{H}^{+}$ions were leaving the membrane. Besides, the standard deviations in the nonpalmitoylated $\mathrm{E}$ protein pentamer are much higher than those in the palmitoylated $\mathrm{E}$ protein pentamer. It means, for each layer, the potentials at different positions are different, which results in non-constant forces acting on cations when the ions approach the membrane. It does not help to repel cations in the cytoplasm. However, there is no significant difference between the non-palmitoylated and palmitoylated $\mathrm{E}$ protein pentamer on the luminal side (N-terminal) (Figure 7B). Although the standard deviations of the palmitoylated $\mathrm{E}$ protein pentamer are higher than those in the nonpalmitoylated $\mathrm{E}$ protein pentamer, they are only one-third of those in the $\mathrm{C}$-terminal for the non-palmitoylated $\mathrm{E}$ protein pentamer. In general, the electrostatic force of the palmitoylated $\mathrm{E}$ protein is more supportive for transferring cations from lumen to cytoplasm.

\section{CONCLUSIONS}

The E protein is an important SARS-CoV-2 structural protein. The pentameric structure of $\mathrm{E}$ protein serves as viroporin. Palmitoylation is a common and important PTM on the E protein. MD simulations are performed on the $\mathrm{E}$ protein pentamers with and without palmitoylation in this study. Based on the MD simulations, the topological and electrostatic studies reveal how palmitoylation affects the $\mathrm{E}$ protein pentamer. Without the palmitoylation, the pentametric structure loses dynamic equilibrium and the pore radius was significantly decreased and the pore collapsed. In the electrostatic studies, the luminal side of the $\mathrm{E}$ protein pentamer is highly negatively charged while the cytosolic side is highly positively charged. The surface potential supports the attraction of cations on the luminal side and the repulsion of cations on the cytosolic side. This finding is consistent with the finding of previous studies that the viroporin prefers cation transfer. The palmitoylation on the $\mathrm{E}$ protein pentamer extends the positively charged area on the cytosolic side and increases the electric interaction with the membrane on the luminal side. Additionally, the PCA reveals that palmitoylation may increase the motility of the $\mathrm{E}$ protein pentamer. In the end, we simulated $\mathrm{H}^{+}$ions approaching the $\mathrm{E}$ protein pentamer on the luminal side and leaving the $\mathrm{E}$ protein on the cytosolic side.
The results indicate that the electrostatic force on the palmitoylated E protein pentamer can provide a constant force along a certain layer, helping cation transfer from the lumen to the cytoplasm. To go further and more accurately prove the ion transferring direction, more sophisticated biological experiments are required. This study reveals the importance of palmitoylation on the $\mathrm{E}$ protein pentamer, which is helpful for the treatment development of COVID-19 and other corona virus-related diseases.

\section{ASSOCIATED CONTENT}

\section{S1 Supporting Information}

The Supporting Information is available free of charge at https://pubs.acs.org/doi/10.1021/acs.jctc.1c00359.

Structure alignment and the sequence alignment of SARS-CoV2 and SARS-CoV; atomic representation of palmitoylation; placement of simulated $\mathrm{H}+$ ions for electrostatic force testing; backbone RMSD of the E protein pentamer with and without palmitoylation; minimal pore radius of the E-protein pentamer in Cterminal and $\mathrm{N}$-terminal; volume of the $\mathrm{E}$ protein pentamer in the $\mathrm{C}$-terminal part; minimal pore radius of the E-protein pentamer in the middle part; RMSF of the residues; distance of the salt bridges; PCA; and rotation of the PCA for the non-palmitoylated and palmitoylated E protein pentamer (PDF)

\section{AUTHOR INFORMATION}

\section{Corresponding Author}

Lin Li - Computational Science Program, The University of Texas at El Paso, El Paso, Texas 79968, United States; Department of Physics, The University of Texas at El Paso, El Paso, Texas 79968, United States; O orcid.org/0000-00016447-2398; Email: 1li5@utep.edu

\section{Authors}

Shengiie Sun - Computational Science Program, The University of Texas at El Paso, El Paso, Texas 79968, United States

Chitra Karki - Computational Science Program, The University of Texas at El Paso, El Paso, Texas 79968, United States

Javier Aguilera - Department of Biological Sciences and Border Biomedical Research Center, The University of Texas at El Paso, El Paso, Texas 79968, United States 
Alan E. Lopez Hernandez - Computational Science Program, The University of Texas at El Paso, El Paso, Texas 79968, United States

Jianjun Sun - Department of Biological Sciences and Border Biomedical Research Center, The University of Texas at El Paso, El Paso, Texas 79968, United States; (1) orcid.org/ 0000-0001-9399-7644

Complete contact information is available at: https://pubs.acs.org/10.1021/acs.jctc.1c00359

\section{Notes}

The authors declare no competing financial interest.

\section{ACKNOWLEDGMENTS}

This work was supported by grant SC1GM132043 from the National Institutes of Health and grant 5U54MD007592 from the National Institutes on Minority Health and Health Disparities, a component of the NIH. The calculations and analyses were performed at the Texas Advanced Computing Center. We thank Mr. Jixuan Pan for writing improvements.

\section{REFERENCES}

(1) (a) Stadler, K.; Masignani, V.; Eickmann, M.; Becker, S.; Abrignani, S.; Klenk, H.-D.; Rappuoli, R. SARS-beginning to understand a new virus. Nat. Rev. Microbiol. 2003, 1, 209-218. (b) De Wit, E.; Van Doremalen, N.; Falzarano, D.; Munster, V. J. SARS and MERS: recent insights into emerging coronaviruses. Nat. Rev. Microbiol. 2016, 14, 523.

(2) (a) Park, J.-E.; Jung, S.; Kim, A.; Park, J.-E. MERS transmission and risk factors: a systematic review. BMC Publ. Health 2018, 18, 574.

(b) World Health Organization. WHO MERS Global Summary and Assessment of Risk; World Health Organization, 2019.

(3) (a) Wu, F.; Zhao, S.; Yu, B.; Chen, Y.-M.; Wang, W.; Song, Z.G.; Hu, Y.; Tao, Z.-W.; Tian, J.-H.; Pei, Y.-Y.; Yuan, M.-L.; Zhang, Y.L.; Dai, F.-H.; Liu, Y.; Wang, Q.-M.; Zheng, J.-J.; Xu, L.; Holmes, E. C.; Zhang, Y.-Z. A new coronavirus associated with human respiratory disease in China. Nature 2020, 579, 265-269. (b) Yang, P.; Wang, X. COVID-19: a new challenge for human beings. Cell. Mol. Immunol. 2020, 17, 555-557.

(4) Khudadad, U.; Safi, N.; Aftab, W.; Ali, A.; Siddiqi, S. The COVID-19 pandemic: an opportunity to strengthen health systems in Afghanistan. East. Mediterr. Health J. 2021, 27, 220.

(5) Madjid, M.; Safavi-Naeini, P.; Solomon, S. D.; Vardeny, O. Potential effects of coronaviruses on the cardiovascular system: a review. JAMA Cardiol. 2020, 5, 831-840.

(6) Hall, K. S.; Samari, G.; Garbers, S.; Casey, S. E.; Diallo, D. D.; Orcutt, M.; Moresky, R. T.; Martinez, M. E.; McGovern, T. Centring sexual and reproductive health and justice in the global COVID-19 response. Lancet 2020, 395, 1175-1177.

(7) Yao, H.; Chen, J.-H.; Xu, Y.-F. Patients with mental health disorders in the COVID-19 epidemic. Lancet Psychiatr. 2020, 7, No. e21.

(8) Moein, S. T.; Hashemian, S. M.; Mansourafshar, B.; KhorramTousi, A.; Tabarsi, P.; Doty, R. L. Smell dysfunction: a biomarker for COVID-19. International Forum of Allergy \& Rhinology; Wiley Online Library, 2020; pp 944-950.

(9) Ramphul, K.; Mejias, S. G. J. C. Coronavirus disease: a review of a new threat to public health. Cureus 2020, 12, No. e7276.

(10) Masters, P. S. The molecular biology of coronaviruses. Adv. Virus Res. 2006, 66, 193-292.

(11) (a) Lan, J.; Ge, J.; Yu, J.; Shan, S.; Zhou, H.; Fan, S.; Zhang, Q.; Shi, X.; Wang, Q.; Zhang, L.; Wang, X. Structure of the SARS-CoV-2 spike receptor-binding domain bound to the ACE2 receptor. Nature 2020, 581, 215-220. (b) Xie, Y.; Karki, C. B.; Du, D.; Li, H.; Wang, J.; Sobitan, A.; Teng, S.; Tang, Q.; Li, L. J. F. i. m. b. Spike proteins of
SARS-CoV and SARS-CoV-2 utilize different mechanisms to bind with human ACE2. Front. Mol. Biosci. 2020, 7, 392.

(12) de Haan, C. A. M.; Rottier, P. J. M. Molecular interactions in the assembly of coronaviruses. Adv. Virus Res. 2005, 64, 165-230.

(13) (a) Schoeman, D.; Fielding, B. C. Coronavirus envelope protein: current knowledge. Virol. J. 2019, 16, 69. (b) Neuman, B. W.; Kiss, G.; Kunding, A. H.; Bhella, D.; Baksh, M. F.; Connelly, S.; Droese, B.; Klaus, J. P.; Makino, S.; Sawicki, S. G.; Siddell, S. G.; Stamou, D. G.; Wilson, I. A.; Kuhn, P.; Buchmeier, M. J. A structural analysis of $\mathrm{M}$ protein in coronavirus assembly and morphology. $J$. Struct. Biol. 2011, 174, 11-22.

(14) Venkatagopalan, P.; Daskalova, S. M.; Lopez, L. A.; Dolezal, K. A.; Hogue, B. G. Coronavirus envelope (E) protein remains at the site of assembly. Virology 2015, 478, 75-85.

(15) (a) Nieva, J. L.; Madan, V.; Carrasco, L. Viroporins: structure and biological functions. Nat. Rev. Microbiol. 2012, 10, 563-574. (b) Nieto-Torres, J. L.; DeDiego, M. L.; Álvarez, E.; JiménezGuardeño, J. M.; Regla-Nava, J. A.; Llorente, M.; Kremer, L.; Shuo, S.; Enjuanes, L. Subcellular location and topology of severe acute respiratory syndrome coronavirus envelope protein. Virology 2011, 415, 69-82.

(16) Westerbeck, J. W.; Machamer, C. E. The infectious bronchitis coronavirus envelope protein alters Golgi $\mathrm{pH}$ to protect the spike protein and promote the release of infectious virus. J. Virol. 2019, 93, No. e00015.

(17) (a) DeDiego, M. L.; Álvarez, E.; Almazán, F.; Rejas, M. T.; Lamirande, E.; Roberts, A.; Shieh, W.-J.; Zaki, S. R.; Subbarao, K.; Enjuanes, L. A severe acute respiratory syndrome coronavirus that lacks the $\mathrm{E}$ gene is attenuated in vitro and in vivo. J. Virol. 2007, 81, 1701-1713. (b) Ortego, J.; Ceriani, J. E.; Patiño, C.; Plana, J.; Enjuanes, L. Absence of $\mathrm{E}$ protein arrests transmissible gastroenteritis coronavirus maturation in the secretory pathway. Virology 2007, 368, 296-308. (c) Curtis, K. M.; Yount, B.; Baric, R. S. Heterologous gene expression from transmissible gastroenteritis virus replicon particles. $J$. Virol. 2002, 76, 1422-1434.

(18) Li, Y.; Surya, W.; Claudine, S.; Torres, J. Structure of a conserved Golgi complex-targeting signal in coronavirus envelope proteins. J. Biol. Chem. 2014, 289, 12535-12549.

(19) Torres, J.; Wang, J.; Parthasarathy, K.; Liu, D. X. The transmembrane oligomers of coronavirus protein E. Biophys. J. 2005, $88,1283-1290$.

(20) (a) Wang, C.; Takeuchi, K.; Pinto, L. H.; Lamb, R. A. Ion channel activity of influenza A virus M2 protein: characterization of the amantadine block. J. Virol. 1993, 67, 5585-5594. (b) Mould, J. A.; Paterson, R. G.; Takeda, M.; Ohigashi, Y.; Venkataraman, P.; Lamb, R. A.; Pinto, L. H. Influenza B virus BM2 protein has ion channel activity that conducts protons across membranes. Dev. Cell 2003, 5, 175-184. (c) Pham, T.; Perry, J. L.; Dosey, T. L.; Delcour, A. H.; Hyser, J. M. J. S. r. The rotavirus NSP4 viroporin domain is a calcium-conducting ion channel. Sci. Rep. 2017, 7, 43487. (d) NietoTorres, J. L.; Verdiá-Báguena, C.; Jimenez-Guardeño, J. M.; ReglaNava, J. A.; Castaño-Rodriguez, C.; Fernandez-Delgado, R.; Torres, J.; Aguilella, V. M.; Enjuanes, L. Severe acute respiratory syndrome coronavirus $\mathrm{E}$ protein transports calcium ions and activates the NLRP3 inflammasome. Virology 2015, 485, 330-339. (e) Wilson, L.; Mckinlay, C.; Gage, P.; Ewart, G. SARS coronavirus E protein forms cation-selective ion channels. Virology 2004, 330, 322-331.

(21) Fung, T. S.; Liu, D. X. Post-translational modifications of coronavirus proteins: roles and function. Future Virol. 2018, 13, 405430.

(22) (a) Chen, S.-C.; Lo, S.-Y.; Ma, H.-C.; Li, H.-C. Expression and membrane integration of SARS-CoV $\mathrm{E}$ protein and its interaction with M protein. Virus Gene. 2009, 38, 365-371. (b) Yuan, Q.; Liao, Y.; Torres, J.; Tam, J. P.; Liu, D. X. Biochemical evidence for the presence of mixed membrane topologies of the severe acute respiratory syndrome coronavirus envelope protein expressed in mammalian cells. FEBS Lett. 2006, 580, 3192-3200.

(23) Liao, Y.; Yuan, Q.; Torres, J.; Tam, J. P.; Liu, D. X. Biochemical and functional characterization of the membrane association and 
membrane permeabilizing activity of the severe acute respiratory syndrome coronavirus envelope protein. Virology 2006, 349, 264275.

(24) (a) Lopez, L. A.; Riffle, A. J.; Pike, S. L.; Gardner, D.; Hogue, B. G. Importance of conserved cysteine residues in the coronavirus envelope protein. J. Virol. 2008, 82, 3000-3010. (b) Boscarino, J. A.; Logan, H. L.; Lacny, J. J.; Gallagher, T. M. Envelope protein palmitoylations are crucial for murine coronavirus assembly. J. Virol. 2008, 82, 2989-2999.

(25) (a) Salaun, C.; Greaves, J.; Chamberlain, L. H. The intracellular dynamic of protein palmitoylation. J. Cell Biol. 2010, 191, 1229-1238.

(b) Fujiwara, Y.; Kondo, H. X.; Shirota, M.; Kobayashi, M.; Takeshita, K.; Nakagawa, A.; Okamura, Y.; Kinoshita, K. J. S. r. Structural basis for the membrane association of ankyrinG via palmitoylation. Sci. Rep. 2016, 6, 23981.

(26) Surya, W.; Li, Y.; Torres, J. Structural model of the SARS coronavirus E channel in LMPG micelles. Biochim. Biophys. Acta Biomembr. 2018, 1860, 1309-1317.

(27) Kim, D. E.; Chivian, D.; Baker, D. J. N. Protein structure prediction and analysis using the Robetta server. Nucleic Acids Res. 2004, 32, W526-W531.

(28) Jo, S.; Kim, T.; Iyer, V. G.; Im, W. CHARMM-GUI: a webbased graphical user interface for CHARMM. J. Comput. Chem. 2008, 29, 1859-1865.

(29) Huang, J.; MacKerell, A. D., Jr. CHARMM36 all-atom additive protein force field: Validation based on comparison to NMR data. J. Comput. Chem. 2013, 34, 2135-2145.

(30) Van Meer, G.; Voelker, D. R.; Feigenson, G. W. Membrane lipids: where they are and how they behave. Nat. Rev. Mol. Cell Biol. 2008, 9, 112-124.

(31) Mackerell, A. D., Jr.; Feig, M.; Brooks, C. L., III Extending the treatment of backbone energetics in protein force fields: Limitations of gas-phase quantum mechanics in reproducing protein conformational distributions in molecular dynamics simulations. J. Comput. Chem. 2004, 25, 1400-1415.

(32) Essmann, U.; Perera, L.; Berkowitz, M. L.; Darden, T.; Lee, H.; Pedersen, L. G. A smooth particle mesh Ewald method. J. Chem. Phys. 1995, 103, 8577-8593.

(33) Phillips, J. C.; Braun, R.; Wang, W.; Gumbart, J.; Tajkhorshid, E.; Villa, E.; Chipot, C.; Skeel, R. D.; Kalé, L.; Schulten, K. Scalable molecular dynamics with NAMD. J. Comput. Chem. 2005, 26, 17811802.

(34) Humphrey, W.; Dalke, A.; Schulten, K. VMD: visual molecular dynamics. J. Mol. Graph. 1996, 14, 33-38.

(35) Van Der Spoel, D.; Lindahl, E.; Hess, B.; Groenhof, G.; Mark, A. E.; Berendsen, H. J. C. GROMACS: fast, flexible, and free. J. Comput. Chem. 2005, 26, 1701-1718.

(36) Smart, O. S.; Neduvelil, J. G.; Wang, X.; Wallace, B. A.; Sansom, M. S. P. HOLE: a program for the analysis of the pore dimensions of ion channel structural models. J. Mol. Graph. 1996, 14, $354-360$.

(37) Li, L.; Li, C.; Sarkar, S.; Zhang, J.; Witham, S.; Zhang, Z.; Wang, L.; Smith, N.; Petukh, M.; Alexov, E. J. B. b. DelPhi: a comprehensive suite for DelPhi software and associated resources. BMC Biophys. 2012, 5, 9.

(38) Dolinsky, T. J.; Czodrowski, P.; Li, H.; Nielsen, J. E.; Jensen, J. H.; Klebe, G.; Baker, N. A. PDB2PQR: expanding and upgrading automated preparation of biomolecular structures for molecular simulations. Nucleic Acids Res. 2007, 35, W522-W525.

(39) Dias, R. P.; Li, L.; Soares, T. A.; Alexov, E. Modeling the electrostatic potential of asymmetric lipopolysaccharide membranes: The MEMPOT algorithm implemented in DelPhi. J. Comput. Chem. 2014, 35, 1418-1429.

(40) Pettersen, E. F.; Goddard, T. D.; Huang, C. C.; Couch, G. S.; Greenblatt, D. M.; Meng, E. C.; Ferrin, T. E. UCSF Chimera-a visualization system for exploratory research and analysis. J. Comput. Chem. 2004, 25, 1605-1612.
(41) Li, L.; Chakravorty, A.; Alexov, E. DelPhiForce, a tool for electrostatic force calculations: Applications to macromolecular binding. J. Comput. Chem. 2017, 38, 584-593.

(42) Ochsenbauer-Jambor, C.; Miller, D. C.; Roberts, C. R.; Rhee, S. S.; Hunter, E. Palmitoylation of the Rous sarcoma virus transmembrane glycoprotein is required for protein stability and virus infectivity. J. Virol. 2001, 75, 11544-11554.

(43) Percherancier, Y.; Planchenault, T.; Valenzuela-Fernandez, A.; Virelizier, J.-L.; Arenzana-Seisdedos, F.; Bachelerie, F. Palmitoylationdependent control of degradation, life span, and membrane expression of the CCR5 receptor. J. Biol. Chem. 2001, 276, 31936-31944.

(44) Noland, C. L.; Gierke, S.; Schnier, P. D.; Murray, J.; Sandoval, W. N.; Sagolla, M.; Dey, A.; Hannoush, R. N.; Fairbrother, W. J.; Cunningham, C. N. Palmitoylation of TEAD transcription factors is required for their stability and function in Hippo pathway signaling. Structure 2016, 24, 179-186. 